\title{
Janela 60 - Simulador para Aprendizagem Tecnológica do Idoso
}

\author{
Ianny C. V. dos Santos, Karoline da P. F de Macêdo, Kevin W. M. da Silva, \\ Henrique M. Granja, Bárbara F. da S. Souza, Nelson I. Oliveira, Isabel D. Nunes, \\ Andreza S. Santos
}

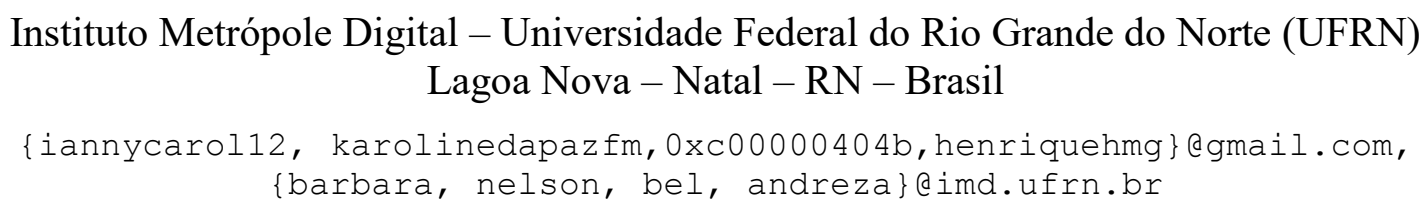

\begin{abstract}
People over 60 are increasingly present in the economic and social activities of our routine, but often, these people did not follow the technological evolution and present difficulties of insertion and updating. This paper have the purpose to present Janela 60 - Simulator for Technological Learning of the Elders. Janela 60 is a online game that allows the senior to simulate the interface of a real program that they can practice without fear of make mistakes. The game allow the old-aged to exercise their cognitive side in relation to memory and the ability to solve problems, as well as to develop their motor coordination.
\end{abstract}

Resumo. As pessoas acima de 60 anos estão cada vez mais presentes nas atividades econômicas e sociais do nosso dia a dia, porém, muitas vezes, essas pessoas não acompanharam a evolução tecnológica e apresentam dificuldade de inserção e atualização. Este artigo tem como objetivo apresentar o Janela 60 - Simulador para Aprendizagem Tecnológica do Idoso. O Janela 60 é um jogo sério online que permite ao idoso simular ações básicas de utilização de um computador sem ter o medo de cometer erros em um ambiente real. As tarefas realizadas permitem que o idoso exercite seu lado cognitivo em relação à memória e à capacidade de resolver problemas, como também desenvolver sua coordenação motora.

\section{Cenário de uso}

As projeções do $\mathrm{IBGE}^{1}$ indicam que $14 \%$ da população brasileira é idosa e esse número deve aumentar ainda mais, tendo em vista que está ocorrendo um declínio significativo nas taxas de fecundidade, ocasionando alterações na pirâmide etária e o aumento contínuo da população idosa [Müller 2012].

Porém, em muitos momentos, somente os pontos negativos do envelhecimento são ressaltados, fazendo com que a maioria dos programas sejam voltados para a diminuição do declínio físico e mental. A informação consegue realizar a difusão veloz e intensa por intermédio de recursos tecnológicos os quais, de acordo com Kachar (2000), promovem a ampliação do acesso ao conhecimento, a qualidade de veiculação e

\footnotetext{
${ }^{1}$ http://biblioteca.ibge.gov.br/index.php/biblioteca-catalogo? view=detalhes\&id=293322
} 
a recepção em diferentes níveis de mídia. Assim, a inclusão digital permite que outro ponto de vista seja considerado, não somente o melhoramento físico e mental, mas também de relacionamento, econômico, social e entretenimento.

Em colaboração a essas percepções, há o aumento da autoestima do idoso com o uso da tecnologia, pois torna-se um sujeito independente e autônomo, diminuindo o estado de depressão e aumentando a sensação de bem-estar [Sales et al. 2014].

Acreditando nisso criou-se um Projeto de Extensão Inclusão Digital para Idosos ProEIDI, no Instituto Metrópole Digital - IMD, da Universidade Federal do Rio Grande do Norte - UFRN, onde foram ministradas aulas de informática básica no computador e no smartphone para o público da terceira idade.

Antes das aulas iniciarem, pesquisas foram realizadas para identificar os principais interesses de aprendizagem dos Idosos [Mariano et al. 2017], mostrando como principal resposta o uso de redes sociais. Porém, durante o curso, foi nítido a grande dificuldade que a maioria possuía em manusear o mouse, usar o teclado e o medo de manipular o computador ou o smartphone e danificá-lo de alguma forma, principalmente por estarem realizando atividades com equipamentos que não conheciam e não lhe pertenciam.

Tal percepção pode ser apoiada pelo trabalho de Raymundo (2013), em que mostra nos resultados de uma pesquisa que $24 \%$ dos idosos possuem medo de utilizar tecnologia e $40 \%$ dos idosos relatam ter receio de danificar o aparelho. Além disso, o ritmo de aprendizagem dos idosos é diferenciado, devendo ser respeitado para diminuir a ansiedade do desconhecido [Fernandes e Ferreira 2012].

Por isso, se fez necessário procurar aplicativos de treinamento das funções do mouse, para melhorar a função motora dos alunos. Foram encontrados aplicativos como SeniorNet ${ }^{2}$, MouseProgram ${ }^{3}$, Let's Go Learn ${ }^{4}$, Tvokids ${ }^{5}$, Agame ${ }^{6}$, Sense-Lang que possibilitaram o desenvolvimento de movimentos como mover o mouse, clicar, dar um clique duplo, selecionar um texto e exercitar o uso do teclado.

A maior dificuldade enfrentada porém, foi de encontrar aplicativos em que essas funções fossem atendidas sem que fossem infantilizadas, além de encontrar um aplicativo que treinasse todas essas funções e fosse em português, para melhor compreensão dos alunos.

Diante dessas situações, houve a necessidade de criar um jogo sério em um ambiente simulado para que os idosos pudessem treinar as funções básicas de um sistema operacional sem se preocupar em errar e danificar o equipamento, visto que o medo que eles possuem é de apagar arquivos, mudar configurações, mudar a organização de arquivos e pastas, fazer download de arquivo com vírus, entre outros. E,

\footnotetext{
2 http://intouch-2000.net/seniornet/sn-exercises.html

${ }^{3} \mathrm{http}: / /$ www.mouseprogram.com/practice.html

${ }^{4} \mathrm{http}: / /$ www.letsgolearn.com/bubble.html

5 http://tvokids.com/preschool/games/bees-and-honey

${ }^{6} \mathrm{http}: / /$ www.agame.com/game/mouse-maze

${ }^{7}$ http://www.sense-lang.org/typing/tutor/EN_lessons.php?lesson=2
} 
VI Congresso Brasileiro de Informática na Educação (CBIE 2017)

Anais dos Workshops do VI Congresso Brasileiro de Informática na Educação (WCBIE 2017)

a partir de tais tarefas, desenvolver suas capacidades motora, de memória e de resolução de problemas.

Os jogos sérios possuem o objetivo de transmitir conteúdo educacional. Segundo Protopsaltis et al (2011), são bastante eficazes como ferramenta de instrução pois apresentam o conteúdo de uma forma alternativa e promovem a motivação e o interesse dos alunos, melhorando a eficácia da aprendizagem. Já os simuladores testam e desenvolvem habilidades do usuário em ambientes que imitam a realidade, permitindo que pessoas realizem tarefas sem sofrerem riscos concretos [Voss, Franzen e Bercht 2016].

O "Janela 60" simula o sistema operacional Windows 10 da Microsoft, o qual é um dos mais usados pela maioria da população no Brasil. Assim, os novos usuários idosos do computador não apenas aprendem funções básicas do Windows, mas também melhoram suas habilidades cognitivas e motoras.

O uso do "Janela 60" apresenta-se flexível devido o seu perfil online, sendo considerado uma ferramenta a ser utilizada em sala de aula como uma prática dentro de uma metodologia ativa, possibilitando que os idosos, em seu primeiro contato com o computador, não tenham medo em utilizá-lo. Além disso, o "Janela 60" pode ser também utilizado fora da sala de aula como ferramenta de prática em qualquer ambiente com acesso a Internet, permitindo aos idosos exercitarem o que estão aprendendo.

\section{Desenvolvimento}

O processo utilizado para implementar o "Janela 60" é o Kanban com algumas características de SCRUM e XP. A necessidade do não uso de sprints fixos deve-se, principalmente, à flexibilização do processo, permitindo que os requisitos sejam projetados, implementados e testados conforme o andamento do processo. O projeto possui uma equipe interdisciplinar com 1 designer, 3 desenvolvedores, 1 aluno pesquisador, 2 pedagogas e 2 professores de Tecnologia da Informação.

A principal característica do SCRUM utilizada, foi a criação de um Product Backlog com os requisitos levantados e o papel de SCRUM Master, em que, um membro da equipe é responsável em organizar e executar o projeto. As práticas de programação em pares e propriedade coletiva foram incorporadas, características encontradas no XP, devido ao grupo ser interdisciplinar e necessitar de um acompanhamento constante da equipe.

A Figura 1 mostra o Diagrama de Casos de Uso com as principais funcionalidades do "Janela 60".

O "Janela 60" permite que os idosos participem de atividades para aprendizado sobre o manuseio do computador, bem como seus periféricos principais, mouse e teclado. Funcionalidades como tirar dúvidas online e o acesso a várias atividades aleatórias do mesmo nível ainda não foram implementadas devido à complexidade da comunicação em tempo real entre o usuário, o tutor e o algoritmo a ser implementado para acesso aleatório às atividades. 
VI Congresso Brasileiro de Informática na Educação (CBIE 2017)

Anais dos Workshops do VI Congresso Brasileiro de Informática na Educação (WCBIE 2017)

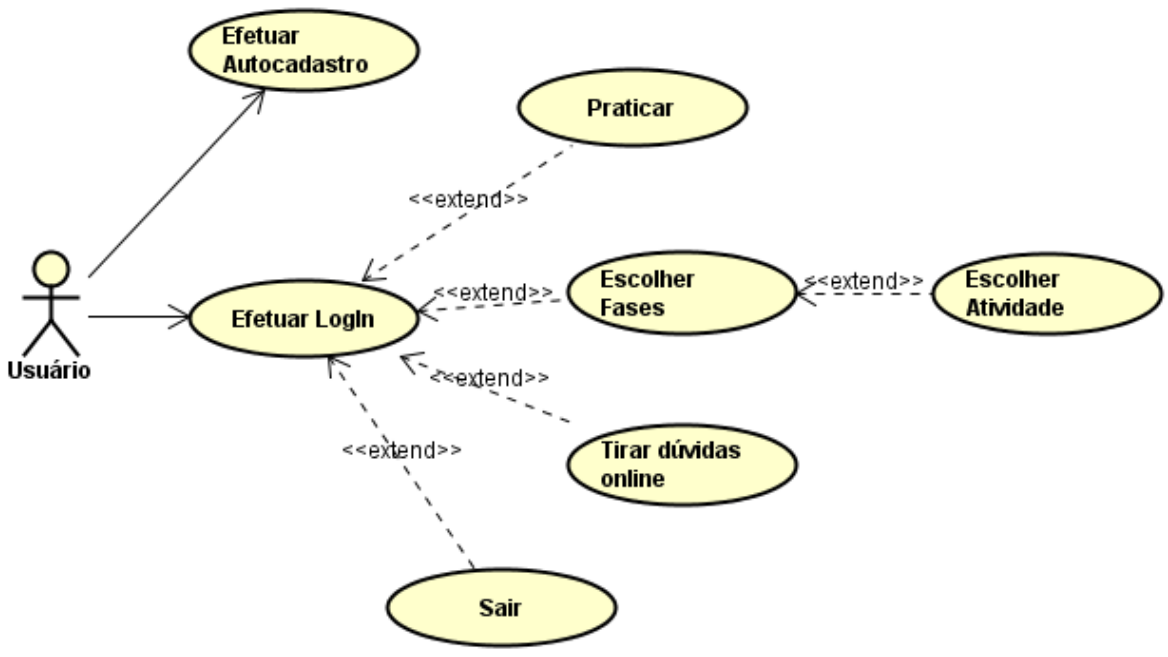

Figura 1. Diagrama de Casos de Uso do "Janela 60"

A arquitetura do "Janela 60" pode ser visualizada na Figura 2. O jogo está sendo desenvolvido para plataforma Web. O front-end é desenvolvido com Construct $2^{8}$, motor de jogo desenvolvido pela Scirra, baseado em programação drag and drop, com ações executadas por eventos. A atividade resultante é exportada para HTML e incorporado ao restante das páginas. $\mathrm{O}$ motor de jogo conta com uma versão gratuita e outra paga.

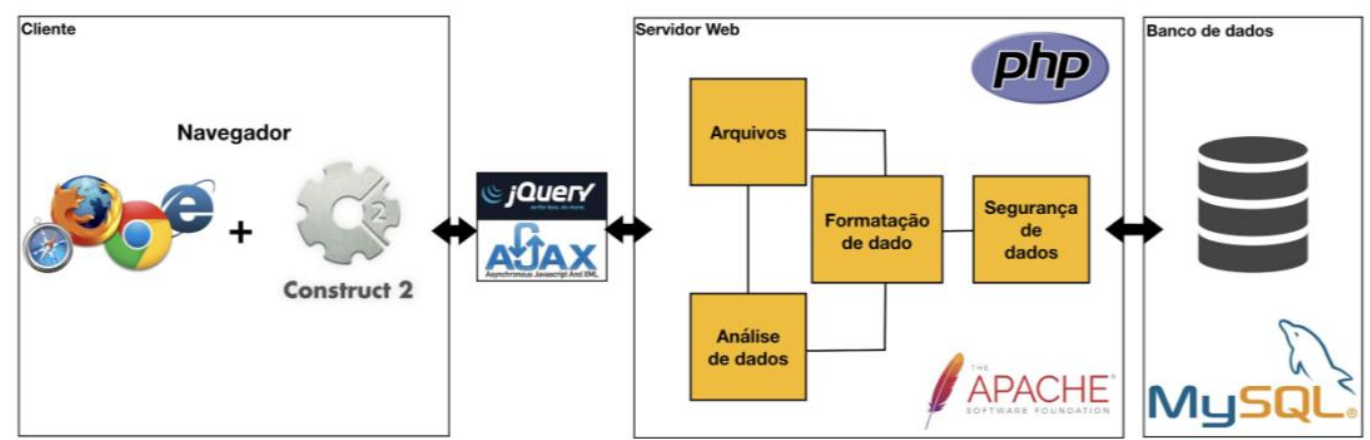

Figura 2. Arquitetura do "Janela 60".

Já o back-end foi desenvolvido em $\mathrm{PHP}^{9}$, que atua integrando as páginas principais com as fases, atividades do jogo e o armazenamento de informações. Para o armazenamento, foi utilizado o MySQL ${ }^{10}$, um banco de dados de estrutura relacional, possibilitando o acompanhamento e armazenamento dos usuários.

Os comandos, visualizações e alterações são realizadas com a linguagem SQL. Os dados manipulados sobre o jogador são: Média de tempo para conclusão de uma

\footnotetext{
${ }^{8}$ https://www.scirra.com/construct2

${ }^{9}$ https://secure.php.net/

10 https://www.mysql.com/
} 
atividade, data e hora das ações, quantidade de tentativas necessárias para concluir a atividade proposta e frequência de acesso. Esses dados são utilizados para melhorar a interação aplicação-usuário. Além dessas informações, também são obtidos feedbacks, através de comentários e dúvidas enviados pelo jogador dentro das próprias atividades.

Para maior segurança das informações inseridas e contidas na aplicação, a "máquina servidor" utilizada para hospedagem, situada no Instituto Metrópole Digital, na Universidade Federal do Rio Grande do Norte, conta com um certificado SSL. As informações que são transitadas entre servidor e cliente são marcadas de acordo com a sensibilidade desta, efetuando a criptografia tanto na comunicação como no conteúdo. Essas medidas aumentam o nível de confiabilidade da aplicação e diminuem as vulnerabilidades para maior disponibilidade do serviço.

Devido ao perfil do público alvo, as páginas principais foram desenvolvidas com recurso de acessibilidade para leitores de tela, possibilitando a inclusão de pessoas com deficiência parcial e/ou total de visão.

A avaliação e validação do jogo não foram realizadas devido ao projeto se encontrar em fase final de desenvolvimento. Releases serão disponibilizadas assim que estiverem funcionais, sob a licença GNU GPL.

\section{Apresentação do Software}

O "Janela 60" possui uma interface condizente com o público alvo a que se destina, considerando um jogo sério, de aprendizagem e voltado para pessoas acima de 60 anos. O jogo está disponível no link https://inclusaodigital.imd.ufrn.br/jogo/login.php.

Inicialmente o usuário deve realizar o login (Figura 3) para ser direcionado ao ambiente do jogo. Caso não tenha login, o usuário pode efetuar seu cadastro acessando no link "Não sou Cadastrado ainda!". Neste passo, é necessário fornecer as seguintes informações (Figura 4): Nome completo, data de nascimento, Sexo, Escolaridade, Endereço com opção de verificador de CEP, e-mail que não esteja cadastrado e uma senha de no mínimo 6 (seis) dígitos. Para a data de nascimento, visando o público alvo da aplicação, o cadastro só aceita usuários acima de 60 anos.

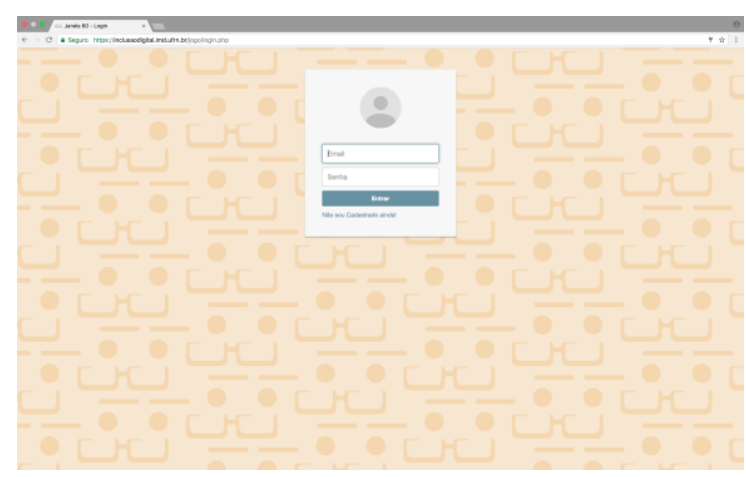

Figura 3 - Tela de Login. 
VI Congresso Brasileiro de Informática na Educação (CBIE 2017)

Anais dos Workshops do VI Congresso Brasileiro de Informática na Educação (WCBIE 2017)

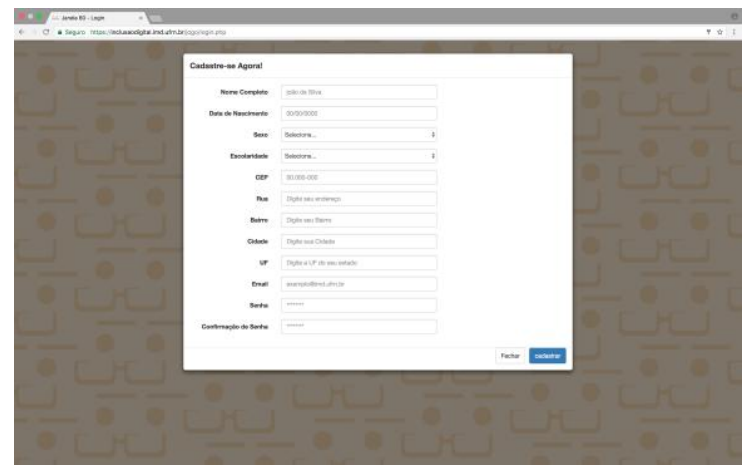

Figura 4 - Área de Cadastro.

Após efetuar o Login, a página principal do jogo (Figura 5) exibe as fases disponíveis, que são liberadas de acordo com o progresso do usuário.

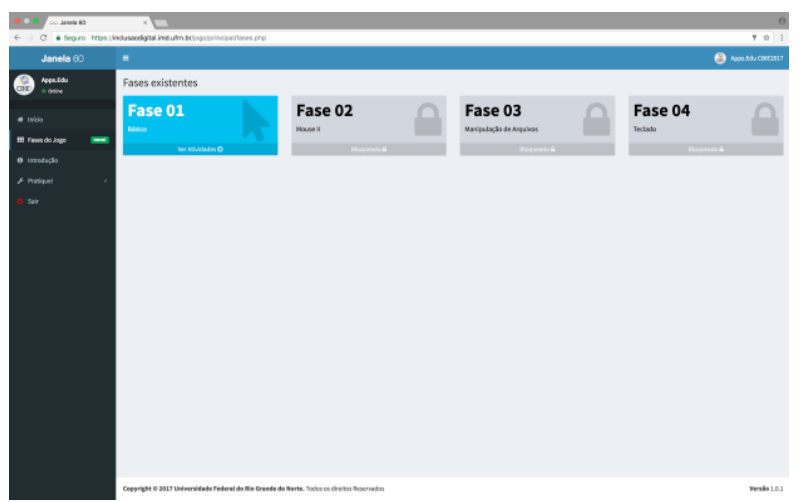

Figura 5 - Tela principal

Na mesma tela da Figura 5, no lado esquerdo, há um Menu com as seguintes opções: "Fases do jogo", local destinado a apresentação das atividades e fases existentes, "Introdução", botão que redireciona a introdução do jogo, caso desejado, "Pratique!", menu multinível que contém mini-jogos para prática do mouse e teclado e o botão "Sair", com função de Logout.

Ao clicar em uma fase que esteja liberada, as atividades existentes aparecem, possibilitando a escolha da atividade desejada. No momento em que o idoso conclui uma atividade, esta é sinalizada para melhor identificação, como mostra a Figura 6.

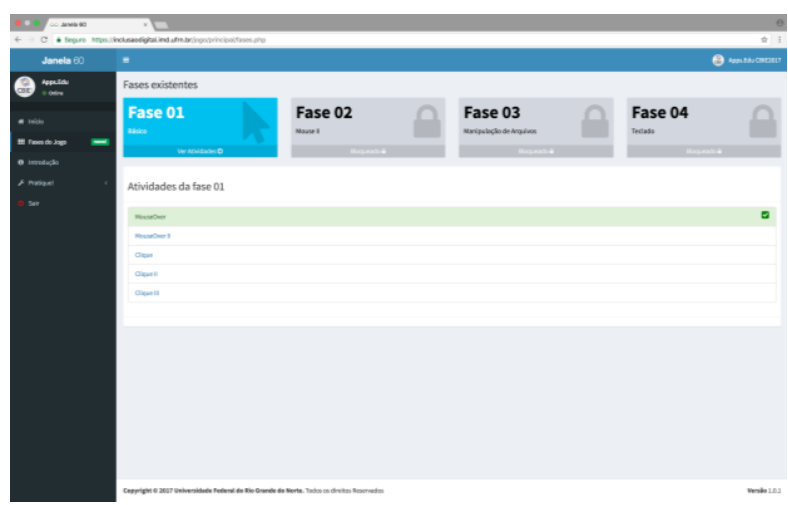

Figura 6 - Fase 1 liberada e atividade 1 feita 
VI Congresso Brasileiro de Informática na Educação (CBIE 2017)

Anais dos Workshops do VI Congresso Brasileiro de Informática na Educação (WCBIE 2017)

Para acessar uma atividade deve-se selecionar dentre as liberadas. Em todas as atividades, o início e o final são marcados por uma animação que passa os objetivos da atividade (Figura 7) e explica sobre as ações e componentes existentes na simulação.

A Figura 7 mostra a atividade "aprender a usar o mouse". Deve-se clicar na "Central de ações", acessar informações da atividade e ter as opções de voltar para a introdução desta, visualizar a resolução e tirar dúvidas/comentar. Além disso, o movimento do mouse também ajuda a desenvolver o lado motor e cognitivo do idoso.
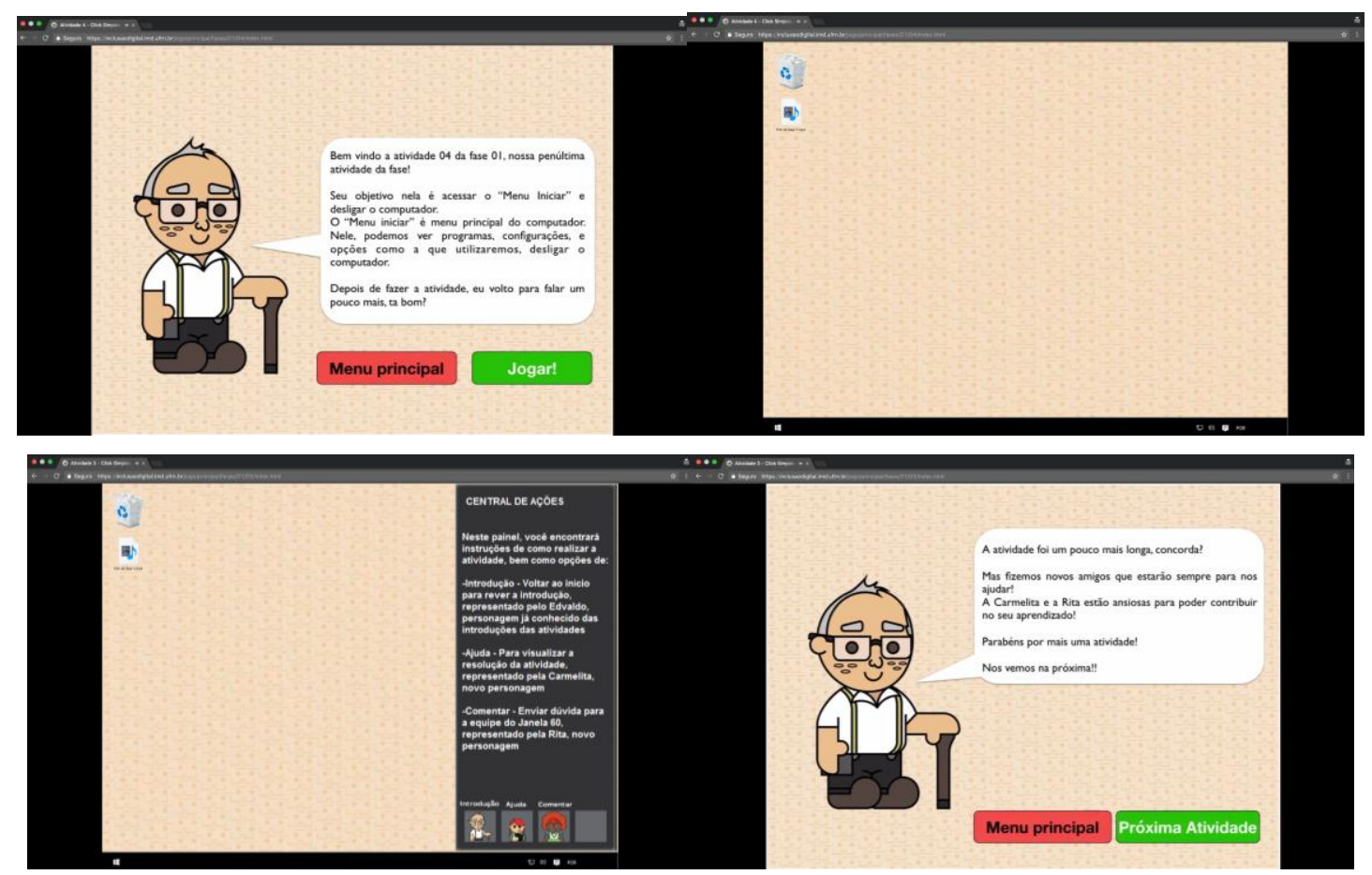

Figura 7 -Exemplo de atividade

Ao finalizar a atividade, o usuário pode ir para a página principal para que continue em sua fase ou escolher em ir para próxima atividades.

\section{Considerações finais}

O “Janela 60" é um jogo sério que simula a utilização do computador, voltado para pessoas acima de 60 anos que não possuem familiaridade com a tecnologia. O jogo permite que os idosos tenham uma prática guiada do uso do computador sem o medo de cometer erros em computadores reais e que desenvolvam seu lado motor e cognitivo.

Algumas funcionalidades ainda estão em desenvolvimento, tais como o acesso aleatório às atividades sem a necessidade de seguir a ordem das fases e a possibilidade de tirar dúvidas online. O objetivo é que o jogo ofereça um tutor inteligente que permita a interação e feedback aos idosos. Outro ponto a ressaltar é a necessidade de constante atualização do sistema operacional do jogo, permitindo que o "Janela 60" esteja sempre atual e atendendo as necessidades reais de uso.

Um dos diferenciais do jogo é permitir que idosos possam praticar o uso de tecnologia em um ambiente direcionado para eles, com tarefas que refletem suas 
dificuldades e medos e que não sejam infantilizadas. Tal característica é reforçada por uma pesquisa realizada pelo Serviço de Proteção ao Crédito (SPC Brasil) e pela Confederação Nacional de Dirigentes Lojistas (CNDL) ${ }^{11}$ em outubro de 2016, a qual mostrou que $37 \%$ dos idosos sentem falta de produtos voltados para eles.

O "Janela 60 " então é um produto que tem forte apelo comercial por ser uma ferramenta de aprendizagem para inserção no mundo tecnológico, sendo de extrema importância nos dias atuais. O mercado para o público idoso está crescendo, formado por um grupo que atinge $14 \%$ da população brasileira e alcançando um poder de compra de 2,4 bilhões ao ano. Assim, tanto os idosos quanto às escolas que oferecem cursos de inclusão digital podem ter o interesse de adquirir o "Janela 60".

\section{Referências Bibliográficas}

Fernandes, F. S. e Ferreira, B. de J. (2012). "Inclusão Digital de Idosos: Um estudo sobre a Realidade do Município de Belém (PA)". Novas Tecnologias na Educação. V. $10 \mathrm{~N}^{\mathrm{o}} 1$.

Kachar, V. (2000). "A terceira idade e o computador: interação e transformações significativas”. A Terceira Idade, São Paulo, v. 11, n. 19, p. 5-21, 2000.

Mariano, L. A., Medeiros, R. L. C. D., Freire, E. P. A. e Nunes, I. D. (2017). "Inclusão Digital para Idosos". II Congresso sobre Tecnologias na Educação - Ctrl+E 2017. Mamanguape/PB.

Müller, D. (2012). “O envelhecimento e a inclusão digital dos idosos”. Monografia de conclusão do Curso de Pedagogia na Universidade Regional Noroeste do Estado do Rio Grande do Sul. Orientador: Hedi Maria Luft. 2012.

Protopsaltis, A., Pannese, L., Papa, D. and Hetzener, S. (2011). "Serious Games and Formal and Informal Learning". eLearning papers. ISSN 1887-1542. n. 25.

Raymundo, T. M. (2013). "Aceitação de Tecnologias por Idosos". Dissertação do Programa de Pós-Graduação Interunidades Bioengenharia - Escola de Engenharia de São Carlos / Faculdade de Medicina de Ribeirão Preto / Instituto de Química de São Carlos da Universidade de São Paulo. 89 p.

Sales, M.B., Mazzali, B.R., Amaral, M.A., Rocha, R.G.O., \& Brito, R. (2014). "Inclusão digital de pessoas idosas: relato de experiências de utilização de software educativo". Revista Kairós Gerontologia, 17(4), pp.63-81. ISSN 1516-2567.

Voss, G. B., Franzen, E. e Bercht, M. (2016). "Explorando a Motivação na Utilização de Mundos Virtuais". V Congresso Brasileiro de Informática na Educação (CBIE 2016), Anais do XXVII Simpósio Brasileiro de Informática na Educação (SBIE 2016).

\footnotetext{
11 http://www.cndl.org.br/noticia/tres-em-cada-dez-idosos-sentem-falta-de-produtos-voltados-para-aterceira-idade-mostra-spc-brasil/
} 